\title{
A Comparative Analysis of Quality of Health Care Delivered in Low and High Task Shifting Environments in Uganda: Implications for Policy
}

\author{
Kanyesigye Rullonga Monicah ${ }^{1,}$, Kaguhangire-Barifaijo Maria ${ }^{1}$, Mohamed Sayeed Bayat ${ }^{2}$ \\ ${ }^{1}$ Uganda Management Institute, Kampala, Uganda \\ ${ }^{2}$ Department of Public Administration, University of Fort Hare and University of Lusaka, Lusaka, Zambia
}

Email address:

mrullonga@gmail.com (K. R. Monicah)

${ }^{*}$ Corresponding author

To cite this article:

Kanyesigye Rullonga Monicah, Kaguhangire-Barifaijo Maria, Mohamed Sayeed Bayat. A Comparative Analysis of Quality of Health Care Delivered in Low and High Task Shifting Environments in Uganda. Implications for Policy. Journal of Human Resource Management. Vol. 8, No. 1, 2020, pp. 1-10. doi: 10.11648/j.jhrm.201200801.11

Received: December 17, 2019; Accepted: December 30, 2019; Published: January 8, 2020

\begin{abstract}
With the increasing global health workers shortage, developing countries like Uganda are embracing task shifting as a form of availing health care amidst the growing population and increasing disease burden. This study examined the quality of healthcare delivered under task shifting in low and high task shifting environments from patients' perspective in Kalangala and Bukomansimbi Districts respectively. An exploratory design was employed with both qualitative and quantitative approaches guided by Banduras theories of social learning and social cognitive. Bivariate analysis was carried out through cross-tabulations on associations between task shifting and quality of healthcare indicators to generate chi-square and p-values. Quality of care was assessed as generally good but much better in high task shifting environments because of the availability of simulations, supervision and mentorship programs which facilitate the health workers to learn even when they possess lower qualifications. The study asserts that good quality healthcare can be provided by virtually any person who is conditioned through training, supervision and mentoring. This has a huge implication for Human Resource for Health (HRH) planning, forecasting and development in the epoch of healthy worker shortage. The study designed and recommended a task shifting model that would facilitate the development of policy framework for task shifting implementation.
\end{abstract}

Keywords: Human Resource for Health, Quality of Healthcare, Task Shifting

\section{Introduction}

The Global scarcity of health workers has affected health care delivery in most developing countries. Currently, the shortage is estimated at 4.3 million health workers. A number of reforms and practices have been considered effective and efficient in order not to interrupt continued service delivery for the growing population [1]. The World Health Organisation (WHO) estimated health worker shortage in Uganda at $80 \%$ in 2016 with a doctor patient ration standing at 1:22,000, and more than a quarter of the world countries having human resource for health shortage challenges. Among the reforms undertaken is the task shifting model; where tasks of highly qualified health workers are shifted and delegated to health workers with lower qualifications $[2,3]$.
This paper presents study findings on a comparative analysis of the quality of care delivered in two task shifting environments one considered high because of the low staffing numbers (Bukomansimbi) and the other as low task shifting because of the high staffing numbers (Kalangala). The paper makes recommendations for human resource planning and development functions based on empirical evidence from patients and how they evaluate the quality of health care they receive based on Bandura's social learning and social cognitive theories [4]. Bandura submitted that individuals can learn and perform as expected if they are conditioned to observation, mentoring, support superivison and have models to show them how things are done. He asserts that in order for this learning to happen, people must have the ability to learn and retain what is being observed, an opportunity to reproduce 
what has been learnt and of course motivated in form of rewards and punishments. These theoretical assumptions insunuate what task shifting would require in order to deliver quality health care in a human resource for healh limited environment. The study interolgated the presence of these theoreticl assumption and resulting quality delivered in these environements.

Task shifting has existed since the $19^{\text {th }}$ century after the French revolution where many institutions were left in a porous state. The French government legitimately acknowledged and frequently employed a class of non-physicians called officiers de santé, who worked in army and navy where there was dire need for medical care services. In china, barefoot doctors were used in rural settings where qualified medical officers were not willing to work; with basic health care training to treat non complicated case such as wound dressing and carrying out immunization services [5-7]. In 1918, the Uganda Health Service (UHS) introduced a cadre between the medical officer and a nurse called medical assistants now referred to as clinical officers and later in 2004, a publication by WHO gave strategies for potential of task shifting, recommending that nurses and clinical officers be trained to deliver primary care for HIV. Eventually, in 2006 WHO picked interest in task shifting as a response to the high shortage of human resource for health critical cadres, coupled with a high burden of infectious diseases, migration of specialists, poor working environments and stumpy motivation specifically in sub Saharan Africa [3].

With a health worker population ration of 1:1298 in Uganda and the staffing situation in the national public sector standing at $68 \%$ for Local government level and $69 \%$ for the national level facilities, the need to increase the number of health workers to match the growing population is evident $[3,8,9]$. Quality of health care is defined as the degree to which health services for individuals and populations increase the likelihood of desired health outcomes, are consistent with current professional knowledge. Quality of health care can be divided into different dimensions according to the aspects of care being assessed. It is the extent to which processes, products, services and relationships are free from defects, constraints and items which do not add value for the customer. Quality of health care is looked at in terms of its safety, patient centered, reliable, timely and equitable to those who receive it [10-12]. Reliable health care is care that is consistent, easily accessible at any time as and when one needs; it whether on weekends, public holidays and with available health workers. This health care must be able to maximize resource use and avoid waste as well. On the other hand, Safety in quality of health care means delivering health care which minimizes risks and harm to service users. Equitable health care is delivering health care which does not vary in quality because of personal characteristics such as gender, race, ethnicity, geographical location or socio economic status. Patient centered health care is care that is considerate of likings and ambitions of individual end users and the cultures of their communities. Lastly, timely health care is care that is geographically reasonable and provided in a setting where skills and resources are appropriate to medical need and in time when needed [ibid].

Ministry of Health in Uganda has categorized operational areas according to their geographical locations. Some places have been categorized as hard to reach and stay; and therefore, difficult to attract health workers [13]. The staffing situation in such places has been reported low, with over $65 \%$ of health workers reported to prefer work in urban settlements. Kalangala and Bukomansimbi districts both located in the Central Region of Uganda are graded as rural settlements with populations of 50,000 and 154,000 respectively $[14,15]$. Kalangala district is located on the shores of Lake Victoria composed of 84 islands of which 64 islands are habitable and only accessible by water/boat transport. The district has a doctor- patient ratio of 3:50,000 with a public health Centre IV and III on the main Island, and Health centre IIs in the 11 islands: the rest of the 57 Islands have no public health care facilities. In 2017, the health care state in Kalangala district was reported worsening because of insufficient health facilities and more so a District Hospital. Many lives were reported lost on boats trying to reach nearby hospitals of Entebbe and Masaka Districts [16-18] and some dying because they can't afford the cost of hiring a boat especially at night. The 2017 Human resource for health audit report indicates that Kalangala has an $82 \%$ staffing levels for its 17 health facilities, which is very good compared to WHO recommended 75+, although the facilities are few and meagerly distributed for the geography of Kalangala and its population [9].

Bukomansimbi district on the other hand is located south of the equator in the central region of Uganda. The district has two Counties (Bukomansimbi South and Bukomansimbi North) with five Sub-Counties (Bigasa, Kitanda, Kibinge, Butenga and Bukomansimbi Town Council). There are 16 health units, 9 belong to government and 7 belong to private. There is one Health centre IV, ten HCIIIs and five HCIIs. The doctor patient ratio is $1: 152,400$, nurse patient ration is $12: 152,400$, clinical officer's patient ration is $10: 152,400$ and midwife patient ration is $9: 152,400$. Eighty percent $(80 \%)$ of the population in Bukomansimbi district depends predominantly on substance farming. Like Kalangala, there is no government hospital in the entire district $[20,15]$.

\section{Method}

The study employed an exploratory design with both qualitative and quantitative approaches of inquiry in localized units of Bukomansimbi and Kalangala Districts with different tasks-shifting levels. Kalangala district is a low task-shifting district with a high level of staffing at $82 \%$, where the recommended staffing is $75 \%$. Bukomansimbi district, on the other hand, has a staffing level of only $62 \%$, and hence is considered a high task-shifting district [9].

The combined study population was estimated to be 150,000: 100,000 for Bukomansimbi district for the 3 parishes of Butenga, Kitanda and Bigasa, and 50,000 of Kalangala district $[15,20]$. The target population was 150 participants 
consisting of health workers in health centre IVs and IIIs and patients receiving care in these facilities. The study used systematic sampling, with semi structured questionnaires on 100 patients in the facilities who are health care consumers and purposive sampling with self-administered questionnaires for 50 health workers. Data was analyzed using themes put into tables with variables on quality of health care pillars. Bivariate analysis was carried out through cross-tabulations on associations between task shifting and quality of health care indicators to generate chi-square and p-values.

\section{Results}

Quality of health care was assessed on the variables of safety, accessibility, equitability, timeliness, and patient centeredness under task shifting in Kalangala and Bukomansimbi districts.

\subsection{Reliability of Healthcare Under Task-shifting}

The aspects of reliable healthcare, included ability to see the same person each time one visits the facility for consistency and follow up of ailments, adequate information given about one's ailment and the treatment prescribed, an opportunity to ask questions and feeling confortable to talk to the healthcare provider about personal health problems.

Hypothesis testing for the mean scores on quality of healthcare delivered in high and low task-shifting environments.

Bivariate analysis

In testing the hypotheses, the quality of healthcare was assessed under five indicators which was the basis for the analysis as indicated in the qualitative responses above. Bivariate analysis was carried out through cross-tabulations on associations between the independent variables and the dependent variables to generate chi-square and $p$-values in the $2 \mathrm{x} n$ tables. The chi-square $\left(\chi^{2}\right)$ and $\mathrm{p}$-values generated were used as appropriate to determine the likelihood of any observed relationships between the dependent and independent variables having occurred by chance alone. As an indicator of quality of healthcare, reliability was assessed using four indicators as indicated in Table 1 below. The study sought to test the following hypothesis:

$H_{1}$ : Reliability as an indicator of quality of healthcare was better in a low task-shifting environment (Kalangala) than in a high task-shifting environment (Bukomansimbi).

Table 1. Chi-square statistics for reliability as an indicator of quality of healthcare.

\begin{tabular}{|c|c|c|c|c|}
\hline \multirow{2}{*}{ Measure of reliability } & \multicolumn{2}{|c|}{ Bukomansimbi } & \multicolumn{2}{|c|}{ Kalangala } \\
\hline & $\chi^{2}$ & p-values & $\chi^{2}$ & p-values \\
\hline Same person attends to me whenever I come for medical care & 5.979 & 0.1127 & 4.982 & 0.1731 \\
\hline Adequate information is given about my condition and treatment prescribed & 17.608 & $0.001 *$ & 1.463 & 0.691 \\
\hline I am given an opportunity to ask questions and I am explained to in simple language & 18.841 & $0.0003 *$ & 2.299 & 2.299 \\
\hline $\begin{array}{l}\text { I am comfortable talking to the healthcare provider about my personal problems related to my health } \\
\text { condition }\end{array}$ & 10.378 & $0.012 *$ & 8.219 & $0.042 *$ \\
\hline
\end{tabular}

With a chi-square of 5.978 and p-value of 0.1127 in Bukomansimbi and a chi-square of 4.982 and p-value of 0.1731 for Kalangala, there was no significant difference between the two districts in the same person attending to clients whenever they went for medical care. On the contrary, there was a difference between provision of adequate information about a client's health condition and treatment prescribed. Clients in Bukomansimbi were more likely to be provided with information $\left(\chi^{2}=17.608\right.$, p-value 0.0005$)$ compared to clients in Kalangala district $\left(\chi^{2}=1.463\right.$, p-value 0.691). In both districts, clients were comfortable talking to the healthcare provider about their personal problems related to their health condition with p-values less than 0.05 (Bukomansimbi p-value 0.012; Kalangala p-value 0.042). Overall, reliability as a measure of quality of healthcare was better in Bukomansimbi compared to Kalangala district. As such, the hypothesis that reliability as an indicator of quality of healthcare was better in a low task-shifting environment (Kalangala) than in a high task-shifting environment (Bukomansimbi) was rejected.

Below are some of the qualitative response that were generated

Some Nurses are rude. They don't even greet you; they want you to quickly tell them your ailment and go. When lines are long, they have no time for us. It becomes very difficult to confide in them. You only tell them what your problem is and they give you medicine and you go. But if you find a kind Nurse, then you can have the courage to discuss with them at length. (A female respondent from Luramba HC III in Kalangala district, 2018)

Another patient from Bukomansimbi had this to say:

Sometimes health workers don't tell you anything about your ailment unless you ask. If you don't ask, they leave you to go, but when you ask they tell. They have many patients to treat, so you also have to be assertive to ask. But when you ask, they also show interest and give you time. (A male respondents from Butenga HC IV in Bukomansimbi district, 2018)

From the above quotes, it is evident that while attitude of health workers is noticed in both districts, in Kalangala, some respondents felt that health workers were over stretched, have to work to serve all, while some are considered rude. In Bukomansimbi on the other hand, some respondents felt that health workers should be prompted in order for them to give information relating to patients' diagnosis. Health workers have no self-drive to give health education unless a patient asks, but when patients ask, they are willing to explain and give in time, for health education.

\subsection{Safety of Healthcare Under Task-shifting}

This segment examined aspects of safety in healthcare delivered by health workers who are task shifting. Questions 
asked were; involvement of patients in decision making about the medication and prescription given, discussion between patients and health workers on prescribed medication, experiencing serious problems from a medical mistake and whether the health workers ask patients any medication-related problems. Respondents were further asked whether they receive enough information about the purpose, benefits and risks of medicines and injections given and whether they received instructions about symptoms to watch for in order to seek further care or treatment.

$\mathrm{H}_{2}$ : Safety as an indicator of quality of healthcare was better in a low task-shifting environment (Kalangala) than in a high task-shifting environment (Bukomansimbi). The test results of the five indicators for assessing safety as a measure of quality of health care are detailed in Table 2.

Table 2. Chi-square statistics for safety as an indicator of quality of healthcare.

\begin{tabular}{llll}
\hline \multirow{2}{*}{ Measure of safety } & Bukomansimbi & & Kalangala \\
\cline { 2 - 4 } & $\chi^{2}$ & p-values & $\chi^{2}$ \\
\hline Had a discussion with care provider & 7.688 & $0.053^{2}$ & 14.650 \\
Ever had a serious medical mistake & 0.642 & 0.725 & 16.332 \\
Always asked about allergies & 12.615 & $0.006^{*}$ & 4.366 \\
Given information on drug risks & 17.060 & $0.0001^{*}$ & $0.0021^{*}$ \\
Instructed about symptoms to watch & 14.904 & $0.0004^{*}$ & 0.2245 \\
\hline
\end{tabular}

With a $\chi^{2}$ of 7.688 and p-value of 0.053 in Bukomansimbi and $\chi^{2}$ of 14.650 and $p$-value of 0.0021 for Kalangala, both districts ensured that clients had a review and discussion with their primary care provider about prescribed medications they were given in the last visit at the health facility. However, clients in Kalangala district had ever experienced a serious problem as a result of a medical mistake made by health workers during the past two years (p-value 0.0003) compared to Bukomansimbi (p-value 0.725).

Bukomansimbi district had clients who had ever been asked about medication-related problems like allergies in their visit to the health facility in the past 2 years $\left(\chi^{2} 12.615\right.$; $p$-value 0.006); were given enough information about the purpose, benefits and risks of the treatments the last time they received an injection ( $\chi^{2} 17.060 ; p$-value 0.0001$)$; and received clear instructions from the practitioner about symptoms to watch for and when to seek further care or treatment $\left(\left(\chi^{2} 14.904\right.\right.$; p-value 0.0004$)$. This was contrary to the results in Kalangala where the respective values were $\left(\chi^{2} 4.366\right.$; $p$-value 0.2245$)$; $\left(\chi^{2}\right.$ 1.719; p-value 0.6327) and ( $\chi^{2} 3.425 ; \mathrm{p}$-value 0.3306$)$. Overall, safety as a measure of quality of health care was better in Bukomansimbi compared to Kalangala district. The hypothesis that safety as an indicator of quality of healthcare was better in a low task-shifting environment (Kalangala) than in a high task-shifting environment (Bukomansimbi) was rejected.

Asked to explain their responses, below are some of the responses patients gave about their evaluation of safety of healthcare received:

There was a Nurse who was in charge of immunization. All children she injected would have sleepless nights and be admitted after two days. She didn't know how to give injections but good enough, they transferred her. Coming for immunization meant admission after two days. We all knew it. (Testimony of a mother in Bufumira HC III in Kalangala District, 2018)

Another patient in Bukomansimbi had this to say:

Sometimes they give you medicine without telling you side effects. If you are allergic to some drugs, you get blisters. I was given an injected in 2014, I got a small blister on the leg and scratched it. It developed into a wound and in 2016, my leg was almost amputated, but I was taken to Masaka hospital where specialized care was given to save my leg. If the Nurse had told me the side effects, I would have sought treatment early but I didn't connect the blister to the drug. Some Nurses are careless (Testimony of a male patient in Bukomansimbi, 2018)

While the respondent in Bukomansimbi suffered as a result of a wrong drug prescribed, the patient in Kalangala thought the Nurse was unprofessional in handling immunization vaccine. This could also be patient ignorance on managing children post immunization and not necessarily the problem of a particular Nurse, but it also speaks volumes on what information Nurses give patients on how to take care of themselves and the side effects of the drugs prescribed.

On a positive note however, one patient in Bukomansimbi indicated that she has always received good information about the healthcare and this is what she had to say:

Although there are no doctors, here, the nurses know what they are doing. Every time they are giving you medicine, they tell you to first eat before you take the medicine and not to drink alcohol. They even ask you if you have ulcers before they give you the tablets. I have no problem with them (A female patient in Bigasa HC III in Bukomansimbi District, 2018)

The above revelation shows that nurses, although not highly qualified as a doctor would be, they possess the required skills to handle the needs of patients. They understand the procedure of prescribing and dispensing drugs. The question would be whether the drugs they dispense are the right drugs for the ailments, but the patients are happy with the services whatever the outcomes.

\subsection{Equitability of Healthcare Under Task-shifting}

Respondents were asked questions that relate to equitability of care received. These included being provided with all drugs required as prescribed, given healthcare without discrimination, availability of clean water for taking drug and health workers following up how patients are doing with the medication prescribed. Respondents were further asked how easy it is to access healthcare in the evenings, over the weekends and on public holidays. They were also asked 
whether they receive outreach services in their communities.

$\mathrm{H}_{3}$ : Equitability as an indicator of quality of healthcare was better in a low task-shifting environment (Kalangala) than in a high task-shifting environment (Bukomansimbi). The test results of the five indicators for assessing equitability as a measure of quality of health care are detailed in Table 3.

Table 3. Chi-square statistics for equitability as an indicator of quality of healthcare.

\begin{tabular}{llll}
\hline \multirow{2}{*}{ Measure of equitability } & Bukomansimbi & & Kalangala \\
\cline { 2 - 4 } & $\boldsymbol{\chi}^{\mathbf{2}}$ & p-values & $\boldsymbol{\chi}^{\mathbf{2}}$ \\
\hline Always received prescribed drugs & 7.493 & $0.0577^{*}$ & 18.630 \\
No discrimination & 4.675 & 0.1972 & 2.152 \\
Clean water to take drugs & 21.331 & $0.0001^{*}$ & $0.0003^{*}$ \\
Follow up & 5.504 & 0.1384 & 0.5414 \\
Healthcare in evenings \& public holidays & 24.769 & $0.0001^{*}$ & 3.242 \\
Community outreach services & 12.022 & $0.0073^{*}$ & 8.281 \\
\hline
\end{tabular}

With a $\chi^{2}$ of 7.493 and p-value of 0.0577 in Bukomansimbi and a $\chi^{2}$ of 18.630 and p-value of 0.0003 for Kalangala, both districts ensured that clients were always provided with all the drugs they require as prescribed. In both districts, some level of discrimination was practiced in providing healthcare services (Bukomansimbi $\chi^{2} 4.675$ and p-value 0.1972; Kalangala $\chi^{2} 42.152$ and p-value 0.5414$)$. Health facilities in Bukomansimbi had clean water when clients needed to take drugs $\left(\chi^{2} 21.331\right.$ and p-value 0.0001$)$ compared to Kalangala. No health workers made follow up to know how clients were performing after prescribing medication in both districts but it was easy to receive care from the health facility in the evening, weekend and public holidays in both districts (Bukomansimbi $\chi^{2} 24.769$ and p-value 0.0001; Kalangala $\chi^{2} 8.281$ and p-value 0.0405). In both districts, the communities received outreach services from the health facilities (Bukomansimbi $\chi^{2} 12.022$ and p-value 0.0073; Kalangala $\chi^{2} 21.34$ and p-value 0.0001).

Overall, equitability as a measure of quality of health care was better in Bukomansimbi compared to Kalangala district. The hypothesis that equitability as an indicator of quality of healthcare was better in a low task-shifting environment (Kalangala) than in a high task-shifting environment (Bukomansimbi) was rejected.

However, respondents were probed on the quantitative responses concerning equitability and below are some of their responses:

Community outreaches have a financial gain for the health works from PHC money, so when opportunity comes, they will work, but these are not often. However, following up patients is only for project patients like HIV patients and mothers. May be those projects are funded. But for other illnesses, follow up is rear. (A female patient from Kitanda HCIII in Bukomansimbi District, 2018)

Another respondent had this to say about the drugs not being available at the facilities:

There is limited supply of drugs by the government and they quickly run out. When we see the boat coming to deliver the drugs we all come to the facility and in two weeks, the health workers will say some drugs are over. They start giving us Panadol tablets only, telling us to go and buy in drug shops (A female respondent at Bufumira HC III in Kalangala district, 2018)

One respondent also had this to say about accessing service delivery over the weekends and public holidays.
Most departments at the facility are either closed or with no health worker to attend to patients on weekends and public holidays especially the laboratory and pharmacy. The staff live in Masaka and Kampala... and come late on Monday because they have to wait for the ferry... You wait for lab staff or pharmacy staff and sometimes you have to come the following day ... (A female respondent at Kalangala HC IV in Kalangala district, 2018)

On a positive note, a respondent in Kalangala had this to say about equitability.

The health workers here have regard for all patients, especially the elderly and pregnant mothers. These ones are given special care which is very good. They treat all patients with respect, no discrimination and when the drugs are available, they will give them to you without asking you for money. After all these are not for sale government drugs (A female respondent at Lulamba HC III in Kalangala district, 2018)

While not all drugs prescribed may be available at the facility, depending on the ailments presented, it is worth noting that, the facilities do their best to take care of the patients with the limited resource available hence providing equitable healthcare. However, the issue of outreaches and follow up of patients seems to be successful for the specialized ailments like HIV and Maternal health which are funded.

\subsection{Patient Centered Healthcare Under Task-shifting}

Respondents were asked questions that relate to patient centeredness as an indicator of quality healthcare. These included attention given to patients' concerns, respect given by the staff, involvement in healthcare decision making process, having helpful discussion between patients and health workers, sensitivity of health workers to culture, ethnicity, spirituality and values of patients, patients given privacy as they receive care and use of a language which patients can understand.

$\mathrm{H}_{4}$ : Patient centeredness as an indicator of quality of healthcare was better in a low task-shifting environment (Kalangala) than in a high task-shifting environment (Bukomansimbi). The test results of the eight indicators for assessing safety as a measure of quality of health care are detailed in Table 4. 
Table 4. Chi-square statistics for patient centeredness as an indicator of quality of healthcare.

\begin{tabular}{lllll}
\hline \multirow{2}{*}{ Measure of patient centeredness } & Bukomansimbi & & Kalangala & p-values \\
\cline { 2 - 5 } & $\chi^{2}$ & p-values & $\chi^{2}$ & $0.006^{*}$ \\
\hline Health concerns listened to & 15.977 & $0.0011^{*}$ & 12.487 & 0.162 \\
Treated me with respect & 16.066 & $0.0011^{*}$ & 5.140 & 0.344 \\
Involved me in drug decision making & 9.021 & $0.029^{*}$ & 3.323 & $0.0003^{*}$ \\
Helpful discussion about my illness & 20.721 & $0.0001^{*}$ & 18.711 & 0.579 \\
Respected for my culture\& values & 11.150 & $0.0109^{*}$ & 1.969 & 0.541 \\
Understandable language & 4.123 & 0.249 & 2.153 & 0.1665 \\
Given privacy & 2.4264 & 0.4887 & 5.0736 & 0.7126 \\
A forum for feedback & 11.6944 & $0.007^{*}$ & 1.3698 & \\
\hline
\end{tabular}

In both districts, the concerns of clients were listened to whenever they sought healthcare services (Bukomansimbi $\chi^{2}$ 15.977 and p-value 0.0011 ; Kalangala $\chi^{2} 12.487$ and p-value 0.006). Equally, clients had helpful discussions with the health workers about their illness (Bukomansimbi $\chi^{2} 20.721$ and p-value 0.0001; Kalangala $\chi^{2} 18.711$ and p-value 0.0003).

Unlike Kalangala, the healthcare providers who attended to clients in Bukomansimbi treated them with respect $\left(\chi^{2} 16.066\right.$ and $\mathrm{p}$-value 0.0011$)$; they involved the clients in decision making before prescribing drugs ( $\chi^{2} 9.021$ and p-value 0.029); respected clients' culture, ethnicity and spiritual values $\left(\chi^{2}\right.$ 11.150 and p-value 0.0109); and there was a forum for feedback and clients' concerns were always taken care of $\left(\chi^{2}\right.$ 11.6944 and p-value 0.007). In both districts, the health workers did not communicate to clients in a language that they understood and clients were not given privacy while in care.

Overall, patient centeredness as a measure of quality of health care was better in Bukomansimbi compared to Kalangala district. The hypothesis that patient centeredness as an indicator of quality of healthcare was better in a low task-shifting environment (Kalangala) than in a high task-shifting environment (Bukomansimbi) was rejected.

Respondents were probed on the above responses and below are quotes from some of the respondents;

"When you write anything in the suggestion box, the staff will create enmity against you and the next time they will make you wait and make you sit for long. I avoid even getting close to that box" (a female patient at Butenga in Bukomansimbi district about the feedback forum, 2018)

Another respondent in Kalangala had this to say:

Some health workers generally don't greet patients, but these are few and it's their character. But generally, they care about us and $i$ have no problem with them (a confession of a male patient from Bufumira HCIII in Kalangala district, 2018)

Not all was negative! A respondent in Bukomansimbi district had this to say:

Some of us are naturally rude and rough that even when we come to facilities we forget that these health workers are providing us a service. The [health workers] really try their best to give us good care. Imagine someone attending to people who are ungrateful, in pain and some with life threatening disease. I highly appreciate and sympathize with them (a male patient at Butenga HC IV in Bukomansimbi, 2018)

In Kalangala, a female patient had this to say on language:

Kalangala has people from all walks of life. We speak different languages and very few of us can ably sustain a conversation in English. Some of the health workers don't even know Luganda. But they try their best to make sure that each one of us is spoken to in their language or at least get someone to interpret. They are very good! They ensure that we speak out and say our problems so that we get the right diagnosis (a female patient in Bufumira HC III in Kalangala, 2018)

Customer care in health service delivery may not compare to business customer care. Where patients have pain and one is expected to satisfy their needs by reducing the pain, the challenge is insurmountable. It is worth noting that in both districts, the statistics show a high percentage of agreement that generally healthcare is patient centered as compared to the rest of the indicators of quality of health care. The patient's comments also indicate that they appreciate the efforts of health workers in providing healthcare amidst the challenges of language barrier, negativity from the patients etc.

\subsection{Timely Healthcare Under Task-shifting}

Time taken by a health worker to respond to inquiries at a facility and the time spent with a health worker while delivering healthcare are crucial in assessing quality of healthcare. Aspects examined on timely health care included sufficient time spent with a health worker, receiving immediate response when an inquiry is made, the facility having a resting area as one waits for care and one being able to receive healthcare within an hour after arrival at the facility.

$H_{5}$ : Timeliness as an indicator of quality of healthcare was better in a low task-shifting environment (Kalangala) than in a high task-shifting environment (Bukomansimbi). The test results of the eight indicators for assessing safety as a measure of quality of health care are detailed in Table 5 . 
Table 5. Chi-square statistics for timeliness as an indicator of quality of healthcare.

\begin{tabular}{|c|c|c|c|c|}
\hline \multirow{2}{*}{ Measure of timeliness } & \multicolumn{2}{|c|}{ Bukomansimbi } & \multicolumn{2}{|c|}{ Kalangala } \\
\hline & $\chi^{2}$ & p-values & $\chi^{2}$ & p-values \\
\hline Sufficient time with health worker & 18.361 & $0.0004 *$ & 7.883 & $0.049 *$ \\
\hline Immediate response for inquiry & 12.180 & $0.002 *$ & 11.228 & $0.004 *$ \\
\hline Waiting place available & 5.433 & 0.143 & 3.159 & 0.368 \\
\hline 1 hour patient waiting time & 20.721 & $0.0001 *$ & 18.770 & $0.0003 *$ \\
\hline
\end{tabular}

From the analysis table 5 above, the time clients spent with the healthcare provider was sufficient in both districts (Bukomansimbi $\chi^{2} 18.361$ and p-value 0.0004; Kalangala $\chi^{2}$ 7.883 and p-value 0.049). Equally, clients got immediate response whenever they made a health inquiry (Bukomansimbi $\chi^{2} 12.180$ and p-value 0.002; Kalangala $\chi^{2}$ 11.228 and $p$-value 0.004). Facilities in both districts did not have a place where clients rested while waiting. In both districts, clients were able to get healthcare within 1 hour of arrival at the facility (Bukomansimbi $\chi^{2} 20.721$ and p-value 0.0001 ; Kalangala $\chi^{2} 18.770$ and p-value 0.0003 ).

Overall, timeliness as a measure of quality of health care was the same in Bukomansimbi and Kalangala districts. The hypothesis that timeliness as an indicator of quality of healthcare was better in a low task-shifting environment (Kalangala) than in a high task-shifting environment (Bukomansimbi) was rejected.

Probed concerning the challenge of timely healthcare, the following responses were noted;

It's a common thing to come here and spend almost the whole morning without a health worker especially on Mondays. They come late because they go away on the weekend. They find so many patients and you have to also wait for long. Such delays are inevitable although they are for particular days. (A male patient from Bufumira HC III in Kalangala District, 2018)

In Bukomansimbi however, the story was different, it was about numbers

In some seasons, many people fall sick and we have very few health workers. If you find like one or two at the facility, you wait for a long time. But when they are all available, especially on Wednesdays when there is immunization, then we take a short time" (a mother from Bigasa HCIII in Bukomansimbi, 2018)

The stretching of health workers who have to look after so many patients cannot be over looked. There are many patients and in known seasons when many sicknesses fall such as when malaria is rampant, the number of health workers is not increased. Patient waiting time therefore can be said to be seasonal.

\section{Discussion}

\subsection{Multivariate Analysis}

To relate the outcome variable with the independent variables, a multiple regression analysis was carried out. The multiple regression equation used was given by the formula: $\mathrm{Y}$ $=\beta_{0}+\beta_{1} \mathrm{X}_{1}+\beta_{2} \mathrm{X}_{2}+\beta_{3} \mathrm{X}_{3}+$--.. Where $\mathrm{Y}$ is the outcome variable i.e. direct costs or indirect costs of health care, $\beta_{0}$ is a constant; $\beta_{1}, \beta_{2}$ and $\beta_{3}$ are regression coefficients of independent variables $X_{1}, X_{2}$ and $X_{3}$ respectively [18]. In making up the model, only factors that were found to be associated with the outcome variable from bivariate analysis and those known to have a bearing on the quality of health care were used. All categorical variables were transformed into dummies to enable performance of linear regression. The best model for each category of reliability, safety, equitability, patient centeredness and timeliness as measurements for quality of health care was selected.

Table 6. Regression statistics for quality of healthcare.

\begin{tabular}{lll}
\hline Variables & t Statistic & P-value \\
\hline Reliability & & \\
Adequate information given & 0.023 & $0.002^{*}$ \\
Opportunity to ask & 0.406 & $0.016^{*}$ \\
Comfortable to confide & 0.713 & $0.079^{*}$ \\
Safety & & \\
Had a discussion with care provider & 2.182 & $0.034^{*}$ \\
Ever had a serious medical mistake & -1.490 & 0.143 \\
Always asked about allergies & 1.451 & 0.153 \\
Given information on drug risks & -0.930 & 0.357 \\
Instructed about symptoms to watch & 0.465 & 0.644 \\
Equitability & & \\
Always received prescribed drugs & -0.158 & 0.875 \\
Clean water to take drugs & -2.370 & $0.022^{*}$ \\
Healthcare in evenings \& public holidays & 0.072 & 0.943 \\
Community outreach services & 0.117 & 0.907 \\
Patient centeredness & & \\
Health concerns listened to & 2.384 & $0.021^{*}$ \\
Treated me with respect & -2.342 & $0.023^{*}$ \\
Involved me in drug decision making & 0.605 & 0.548 \\
Helpful discussion about my illness & 0.325 & 0.747 \\
Respected for my culture\& values & -1.909 & 0.062 \\
A forum for feedback & 0.787 & 0.435 \\
Timeliness & & \\
Sufficient time with health worker & -0.766 & 0.447 \\
Immediate response for inquiry & 0.761 & 0.451 \\
1 hour patient waiting time & -0.150 & 0.881 \\
\hline
\end{tabular}

Dependent variable: Quality of health care. $(* \mathrm{p}<0.05)$ Adjusted R Square $=$ 0.64

From the regression analysis in Table 6, the results show an adjusted $\mathrm{R}$ square (proportion of the variability) of 0.64 . It implies that the model has good explanatory power i.e. it explains up to $64 \%$ of the variability of the variables that are associated with quality of healthcare services in low and high task shifting environments. Consequently, the quality of healthcare was influenced by the following factors which had a positive correlation. Adequacy of information given to clients, the opportunity to ask and comfortable to confide under reliability, influenced the quality of health care. With respect to safety, the quality of health care was influenced by the ability of healthcare workers to discuss with the clients. In terms of equitability, 
availability of clean water to take drugs influenced the quality of health care services. Lastly, patient centeredness was influenced by the ability of health workers to listen to the concerns of clients and being able to treat them well.

Whereas Kalangala district is a low task-shifting environment with more health workers at $82 \%$ as compared to Bukomansimbi at $62 \%$, [13] the findings indicated that in most aspects of quality of healthcare, Bukomansimbi scored better than Kalangala.

On the aspect of reliability, there was no significant difference between the two districts in the same person attending to clients whenever they went for medical care. On the contrary, there was a difference between provision of adequate information about a client's health condition and treatment prescribed; Bukomansimbi was doing better than Kalangala. In both districts, clients were comfortable talking to the healthcare provider about their personal problems related to their health condition. These results can be attributed to the trainings, regular support supervision and In-service and mentorship available in Bukomansimbi that healthcare is much more reliable than in Kalangala. There is also a possibility that the personnel in Bukomansimbi district (health workers) are more professional in handling their patients than Kalangala hence their high score in reliability.

Concerning safety as an indicator of quality of healthcare, the findings indicate that both districts ensured that clients had a review and discussion with their primary care provider about prescribed medications they were given in the last visit at the health facility. However, clients in Kalangala district had ever experienced a serious problem as a result of a medical mistake made by health workers during the past two years (p-value 0.0003) compared to Bukomansimbi ( $p$-value 0.725). Respect for clients' values was equally high in Bukomansimbi as compared to Kalangala. While Bukomansimbi scored better than Kalangala in safety, the level of difference was not very significant.

Kalangala district has more facilities than Bukomansimbi district, is much more developed and older district [16], with more health workers, fewer population as compared to Bukomansimbi, the time patients spend at the facility should be less. The findings however indicate the contrary in terms of quality of healthcare delivered. In a study by Musisi, it was noted that a number of health workers take study leave and facilities are left porous without health workers, yet while the statistics show that the staffing is high: the actual numbers in place are few [19].

Interestingly, studies carried out in Sub saharan Africa have found that quality of healthcare under task-shifting was not any different from that where professionals were involved both in results and cost $[12,22-24,26]$. Thompson and Strickland on strategic management advise that an organization's core competencies reside in its people and in its intellectual capital, not in its assets on the balance sheet [24]. This is in support of the study findings that the facilities in Bukomansimbi are depending on the intellectual capital of the few health workers available and not necessarily the numbers.
Moreover, the results of this study can be compared to the study that was carried out in Mozambique [25] where it was found out that surgically trained Clinical Officers produced similar patient outcomes at a significant lower cost as compared to physicians' obstetricians and gynaecologists. While the contradiction indicated safety concerns with HIV/AIDs care in sub Saharan African while analysing task-shifting, [25], this study indicated that patients evaluated healthcare as safe in Bukomansimbi more than in Kalangala. Clients in Kalangala district noted to have ever experienced a serious problem as a result of a medical mistake made by health workers during the past two years (p-value 0.0003) compared to Bukomansimbi (p-value 0.725).

In triangulating the qualitative results, patients in Bukomansimbi appreciated the healthcare provided by male nurses whom they perceived as doctors. While this can be a stereotype kind of thinking that certain roles are better provided by a given gender orientation, there is a possibility that this can be a confounder for the results of the study.

\subsection{Theoretical and Conceptual Connections with the Study Findings}

Bandura's social learning theory gives the study a thought process of looking at what can be done by individuals when given an opportunity to do it regardless of whether they have studied it as long as they are given step by step guidance in doing it. We note that workers in both districts are distinctly performing well and the recipients of services are happy with what they get. This is the connection of the study findings and the theory. However, the theory helps to identify missing links in the performance of health workers under task-shifting. There is limited motivation in form of rewards and punishments. Models/mentors are almost not available to demonstrate to health workers what they are supposed to do. The theory guides that there should be mentorship, and the study findings indicate that mentorship is very limited in both districts although Bukomansimbi is better than Kalangala.

Interestingly, the conceptual aspects of the independent variable were all observed in the task-shifting process in both districts however, the intervening variables that could influence the quality of healthcare were in a limited stream. While tasks are shifted in the conceptual four levels of doctor to Clinical officiers, Clinical Officer to Nurse/Midwives, Nurse to Nursing Assistant and Nursing Assistant to care take/patient. There are limitations in resources, supporting policies, and the much desirable support supervision. The theory though, does not provide aspects of how desired outcomes are evaluated and appraised to base the outcomes on the theory assumptions. The study was able to identify that the theory is limited in areas of evaluation of outcomes of individuals who are being mentored. What methods are in place to know that one's performance is a result of what has been taught through observation, mentoring, supervision and remuneration other than an individual's own initiative to learn and or intrinsic motivation and personal skills? In a place where there is more than one person, how do you 
attribute the performance to all supposing; the effort belongs to one person? Such aspects are not provided for in the theory and the researcher felt that this is a good area of further discourse. Where task-shifting is successful, and mentors are rightfully available, the theory does not provide how one can appraise the outcomes on mentorship without consideration of aspects such as intrinsic motivation of the workers.

\section{Conclusion}

The study concludes that the success story of task-shifting cannot be complete without supervision, mentorship and training. As a way of enhancing task-shifting implementation, it is suiting to say that these aspects were available in both districts for the health workers at different levels. Both districts scored well in patient centered, safe and reliable healthcare although scoring low on equitability of health care. In all aspects of quality of healthcare, one can conclude that all the aspects are well taken care of in both districts although at different levels. However, the study concludes that quality of healthcare is better in Bukomansimbi district than Kalangala although Kalangala district has more health workers than Bukomansimbi. The conclusion is that while numbers are critical, quality delivered can still be achieved with fewer human resources as long as they are conditioned to supervision, in-service training and mentorships.

Theoretically, one can conclude that Bandura's learning theory can be localized to aspects of staff performance only if we have mentors, models, motivation and support supervision in place like they were noted in Bukomansimbi as opposed to Kalangala district. The theory though is not exhaustive in all contexts.

The study recommends that in order to achieve quality healthcare, workers should be motivated, mentored, trained and supervised. There should be regular support supervision in health facilities both from the district and the local communities and empower the patients to demand for quality services. Health workers should be given regular training and mentorship in order for them to learn and be update with medical changes. The training must be systematic and based on skills needs assessment.

With respect to reliability, areas of concern and emphasis should be information given to patients about their ailments, giving patients an opportunity to ask questions. Under safety, emphasis should be on giving information to clients on the risks of drugs prescribed and what the drugs are intended to treat. In terms of equitability, clean water to take drugs was expressed as a need specifically for Kalangala yet both districts were performing well in all other aspects. Under patient centered care, it was noted that patients expressed need for respect, involvement in decisions for treatment plan and respect for their cultures and values. Lastly, under timeliness it was noted that both districts do not have comfortable waiting areas for patients.

Furthermore, whereas task-shifting is a reality in Uganda and perhaps in the rest of Africa, it needs to be recognized, formalized, and mainstreamed into health services policy to address the shortage of $\mathrm{HRH}$. This will require a number of reforms in governance, recruitment and sustainability and a review of the current staffing and health staff cadre levels. It will require harmonization of labor laws and policies. All the changes should then be reflected in the HRH policy and plans of the country. The gist of the policy should not be to limit the growth of health staff numbers but empower lower cadre staff with more skills to carry out more roles in health service delivery. This will be handy in places where higher cadres, such as doctors and senior Nurses, are not available. With this the remuneration and motivation of the cadres should also come automatically.

Importantly, the study recommends a model of task shifting implementation that puts in consideration of the respondents/patients concerns, the health workers expectations and study findings. The recommended model is presented below;

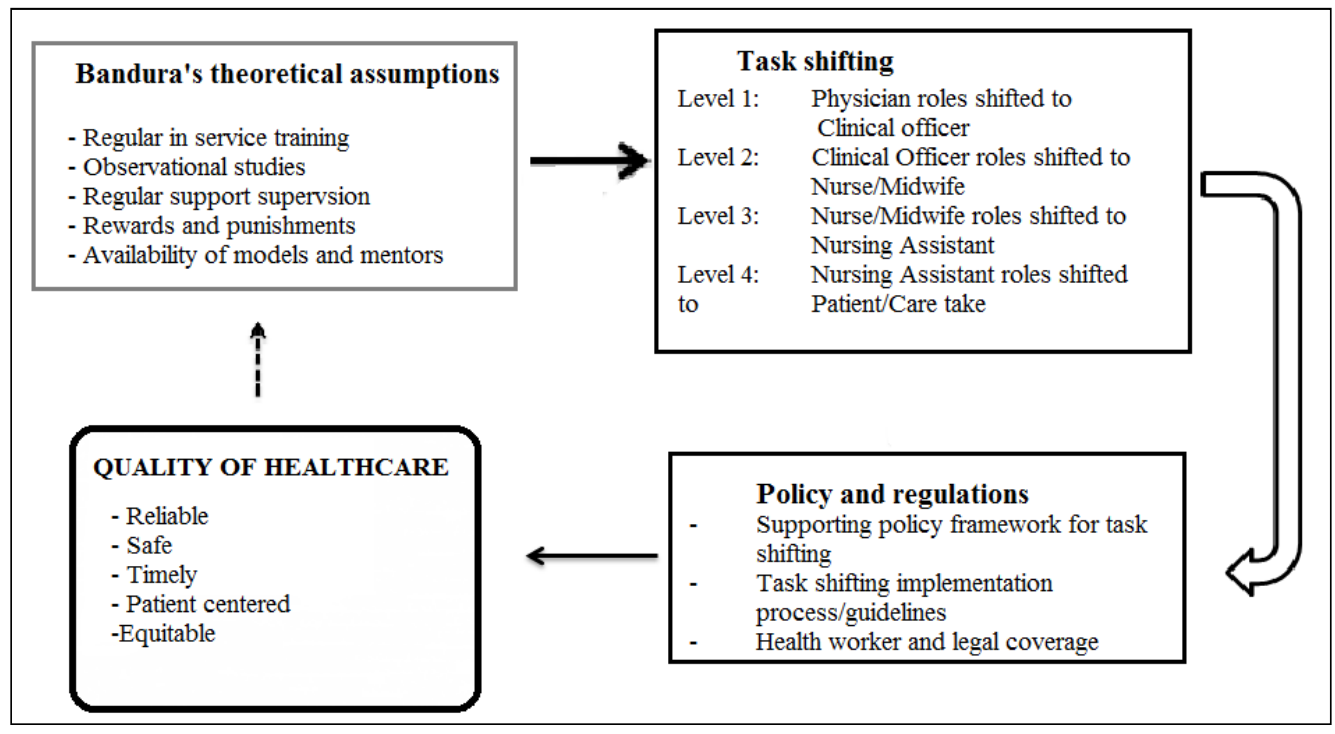

Figure 1. Showing a proposed model for task shifting implementation in health care delivery. 
The recommended task shifting model above starts with theoretical assumptions as expounded by Bandura's theories on social learning and social cognitive. It proposes that there should be a strategy for training health workers while in service in order to avoid dependency on their basic training which is not appropriate for the delegated roles. With mentors and regular support supervision, the model suggests that the four levels of task shifting can now start. These are followed up with regulations and policy support that specifies the guidelines for task shifting, the health worker indemnity and reward and punishment strategies for desired performance. In the end, all the above in place should lead to the desired quality of health care that is safe, reliable, patient centered, timely and equitable.

\section{References}

[1] WHO. (2013, November). Global Health Workforce shortage to reach 12.9 million in coming decades. Retrieved March 18, 2018 , from http://www.who.int/medicenter/new/releases/2013/health-wor kforce-shortage/en/

[2] WHO. (2007, March). Task shifting to tackle health workers shortage. Retrieved 2019 18, February, from http://www.who.int/healthsystem/taskshifting-booklet.pdf

[3] WHO. (2004, March). Integrated management of adolescents and adult illness. Retrieved June 12, 2018, from A 3by5 initiative: www.who.int/3by5/publications/document/imai/eb/treat3milli onpeopleby 2005

[4] Bandura, A. (1977). Social Learning theory. Engelewoods Cliffs: Prentice Hall, Inc.

[5] Heller, R. (1978). Officiers de sante': The second class doctors of nineteenth century France. Medical History, 22 (1), 25-43.

[6] Sidel, V. (1972). The barefoot doctors of the people's Republic of China. New England journal of medicine, 1292-13000.

[7] Xu, S., \& Hu, D. (2017). Barefoot doctors \& the "health care revolution" in rural China. A study centered on Shandong province. Endavour, 136-145.

[8] Ministry of Health. (2014). Annual health Sector Performance Report. Kampala: Government of Uganda.

[9] Ministry of Heath. (2012). Human Resource Audit report 2011: Ministry of health Motivation and Retention strategy 2010/11-2014/15. Kampala: Ministry of Health.

[10] Mainz, J. (2003). Defining and classifying clinical indicators of quality improvement. International Classification of quality of health care, 523-530.

[11] Mildred, G., Lesilie, T., Don a, A., \& Chris, J. (2013). What
Management and Quality Theories are Best for Small Businesses? Jounral of Management and Marketing Research.

[12] Shumbusho, F., Turate, I., Price, J., Lowrance, D., \& Binagwaho, A. (2008). Task shifting to achieve universal acces to HIV care: evaluation of a pilot program of Antiretroviral treatment service delivery by nurses in Rwanda. HIV/AIDS Implementers meeting. Kampala.

[13] Ministry of Health. (2016). Human Resource for Health Audit report. Kampala: Republic of Uganda.

[14] Uganda Bureau of Statistics. (2015). Bukomansimbi Local Government Statistical Abstract. Kampala: Republic of Uganda.

[15] Uganda Bureau of Statistics. (2016). 2014 Statistical Abstract. Kampala: Uganda Bureau of statistics.

[16] BigEye. (2015, June 23). Retrieved November 28, 2015, from http://bigeye.ug/kalangala-district-receives-free-health-service s/\#sthash.wbgxjc4b.dpuf

[17] Bindhe, E. (2012, Mar 29). Kalangala Health Centres Abandoned. Kalangala, Uganda.

[18] Amin, A. (2005). Social Sciences Research, Conception, methodology and analysis. Kampala: Makerere University Printery.

[19] Musisi, N. N. (2017, August 10). The deteriorating state of health care in Kalangala district. Retrieved February 01, 2018, from https://www.cehud.org

[20] Bukomansimbi District Local Government and Uganda Bureau of statistics. (2016). Bukomansimbi District Local Government Statistical Abstract. Bukomansimbi: Governement of Uganda.

[21] UBOS. (2018). 2018 Statistical Abstract. Kampala: Uganda Bureau of Stastistics.

[22] Jouquet, G., Bemelmans, M., Massaquo, M., Arnould, L., Mwagomba, B., Beuernfeind, A., et al. (2009). Cost Analysis of an ARV care programme reaching universial access in Thyolo, Malawi. the IAS Conference on HIV Pathogens, Treatment and Prevention. Cape Town: TUAD 105.

[23] Olikira, B., \& Kasangaki, A. (2014). A scoping study on task shifting; the case of Uganda. BMC Health Services Research, 14: 184 .

[24] Thompson, A., \& Strickland, A. (2004). Strategic management: concepts and cases. Singapore: McGraw -Hill Irwin.

[25] Fulton, D., Scheffler, M., Sparkes, P., Auh, Y., Vujicic, M., \& Soucat, A. (2011). Health workforce skills mix and task shifting in low income countries: a review of recent evidence. BioMed Central.

[26] Olikira, B., Bugisha Beine, E., Kasangaki, A., \& Mugisha, B. M. (2018). Task shifting in health service delivery from a decision \& policy makers perspective: a case of Uganda. Bio Med Central, 284-7. 